\title{
Habilidades sociais de familiares cuidadores de pacientes psiquiátricos
}

\author{
Social skills of family caregivers \\ of psychiatric patients
}

\author{
Diego Costa LIMA ${ }^{1}$ \\ Marina BANDEIRA ${ }^{2}$ \\ Marcos Santos de OLIVEIRA ${ }^{3}$ \\ Joanna Gonçalves de Andrade TOSTES²
}

\section{Resumo}

Com a desinstitucionalização psiquiátrica, as famílias passaram a ter papel fundamental na reinserção social dos pacientes, bem como um sentimento de sobrecarga com esse papel. Um bom relacionamento entre familiares e pacientes constitui um importante fator modulador que pode diminuir a sobrecarga. Entretanto, poucos estudos avaliaram as habilidades sociais dos familiares cuidadores, embora elas sejam necessárias para o desenvolvimento de um bom relacionamento com o paciente. O objetivo desta pesquisa foi avaliar essas habilidades. Foram entrevistados 53 familiares, com aplicação dos instrumentos: Inventário de Habilidades Sociais, Rathus Assertiveness Schedule e questionário sociodemográfico. Os resultados mostraram porcentagem elevada de familiares com baixos escores de habilidades sociais e da subclasse de assertividade. Esses resultados sugerem a necessidade de serem implantados programas de treinamento de habilidades sociais nos serviços de saúde mental, para que os familiares cuidadores desenvolvam um melhor relacionamento com o paciente, potencializando a prestação de cuidados mais adequados.

Palavras-chave: Cuidadores; Habilidades sociais; Pacientes psiquiátricos.

\begin{abstract}
Psychiatric deinstitutionalization has resulted in the greater involvement of family caregivers in patients' social reinsertion as well as the feeling of being overburdened. A good relationship between caregivers and patients is considered to be an important moderating factor that can reduce the burden on families. However, few studies have evaluated the repertoire of caregivers' social skills even though they are the basis for the development of a good relationship. The aim of the present study was to evaluate these skills. Data were collected from 53 caregivers, with the application of the following instruments: Inventory of Social Skills, Rathus Assertiveness Schedule and a sociodemographic

$\boldsymbol{v} \mathbf{v} \boldsymbol{v}$

1 Centro Universitário de Lavras, Departamento de Psicologia, Curso de Psicologia. R. Constância Reis, 99A, Retiro, 37200-000, Lavras, MG, Brasil. Correspondência para/Correspondence to: D.C. LIMA. E-mail: <diegopsicologia@yahoo.com.br>.

2 Universidade Federal de São João Del-Rei, Departamento de Psicologia, Curso de Psicologia. São João Del Rei, MG, Brasil.

3 Universidade Federal de São João Del-Rei, Departamento de Matemática, Curso de Matemática. São João Del Rei, MG, Brasil.

Artigo elaborado a partir da dissertação de D.C. LIMA, intitulada "Habilidades sociais de familiares cuidadores de pacientes com transtornos psiquiátricos". Universidade Federal de São João del-Rei, 2013.
\end{abstract}


questionnaire. Results showed a high percentage of family caregivers with low scores in social skills as well as in assertiveness. These results pointed to the need for implementing social skills training programs in mental health services, so that caregivers can develop a better relationship with patients and consequently be able to provide a better quality of care.

Keywords: Caregivers; Social skills; Psychiatric patients

Os transtornos psiquiátricos têm sido alvo de preocupação da Organização Mundial de Saúde (OMS), devido às suas altas taxas de prevalência e ao ônus gerado para os pacientes e suas famílias (OMS, 2001, 2008). De acordo com Thornicroft e Tansella (2010), pesquisas realizadas em domicílios, entre os anos de 2001 e 2003, em quarenta países, com mais de 60 mil adultos, indicaram que a prevalência de todos os transtornos psiquiátricos, na maioria dos países, variava entre 9,1 e 16,1\%. Segundo dados da OMS (2008), no ano de 2002, os transtornos psiquiátricos foram responsáveis por $13,0 \%$ da carga mundial de doenças. Esse índice foi calculado em termos de dois componentes: anos de vida perdidos por morte prematura e anos vividos com incapacidade. Considerando apenas o segundo componente no cálculo da carga de doenças, os transtornos psiquiátricos foram responsáveis por $31,0 \%$ de todos os anos vividos com incapacidade.

Os transtornos psiquiátricos geram um ônus para os pacientes e seus familiares, em termos de custos monetários e não monetários. Os custos monetários são aqueles relacionados à perda de emprego, redução de produtividade e gastos com prestação de serviços sociais e de saúde, dentre outros (OMS, 2001). Quanto aos custos não monetários, os pacientes sofrem não somente as consequências diretas da doença, mas também o comprometimento de seu funcionamento social, profissional e familiar. Por outro lado, os familiares cuidadores desses pacientes passam por alterações em sua rotina e em sua vida social e profissional, que resultam em sobrecarga e stress advindos do processo de cuidar, provocando sintomas de ansiedade e depressão que afetam sua própria saúde mental (OMS, 2001, 2008; Tessler \& Gamache, 2000; Thornicroft \& Tansela, 2010).

Uma das dificuldades enfrentadas pelos pacientes psiquiátricos, em sua reinserção na comunidade, consiste na emissão de comportamentos problemáticos em interações sociais. Comportamentos como agitação, agressividade física e verbal, falta de higiene, comportamento sexual inadequado, recusa de medicação, abuso de álcool e drogas, dentre outros, têm sido apontados na literatura como fontes frequentes de problemas nas relações sociais dos pacientes. Esses comportamentos problemáticos têm sido alvo de diversas pesquisas avaliativas (Abelha et al., 2006; Bandeira, 2003; Patterson \& Mausbach, 2010; Vidal, Bandeira, \& Gontijo, 2007) ou de intervenções, por meio de treinamento de habilidades de vida cotidiana e de interação social, necessárias à vida dos pacientes na comunidade (Bartels et al., 2004; Trieman \& Leff, 2002). Em consequência desses problemas, os familiares cuidadores passam por situações sociais que requerem negociações complexas com os pacientes e demandam um manejo adequado do seu comportamento. A dificuldade em lidar com os comportamentos problemáticos dos pacientes tem sido citada como um dos principais fatores associados à sobrecarga sentida pelos familiares cuidadores (Barroso, Bandeira, \& Nascimento, 2007; Loukissa, 1995; Maurin \& Boyd, 1990; Rose, 1996; Soares \& Munari, 2007)

Segundo o modelo teórico explicativo da sobrecarga subjetiva dos familiares, elaborado por Maurin e Boyd (1990), algumas variáveis podem diminuir esse grau de sobrecarga, tais como a qualidade do relacionamento com o paciente, o suporte social recebido e as estratégias de enfrentamento utilizadas. Para que esses fatores moduladores atuem, reduzindo a sobrecarga dos familiares, é necessário que estes adquiram um repertório eficiente de habilidades sociais.

As habilidades sociais se referem às diferentes classes de comportamentos, emitidos pelo indivíduo, para lidar de forma adequada com as outras pessoas, em uma interação social. Uma de suas subclasses consiste na assertividade, que se refere à expressão direta e honesta das opiniões e 
sentimentos, assim como defesa dos próprios direitos, sem desrespeitar as necessidades do outro (Z.A.P. Del Prette \& Del Prette, 1999, 2001). Essas habilidades são importantes para que os familiares tenham condições de lidar com os comportamentos do paciente nas situações da vida cotidiana. Um repertório adequado de habilidades sociais pode, ainda, permitir que os familiares atuem como agentes educativos junto ao paciente, termo atribuído a qualquer pessoa que promova regularmente o processo de aprendizagem e/ou desenvolvimento de outras (Del Prette \& Del Prette, 2008). Repertórios adequados de habilidades sociais possibilitam o desenvolvimento de melhor relacionamento com os pacientes e a busca mais efetiva de suporte social, diminuindo a sobrecarga, o que resulta em melhor atendimento ao paciente na vida cotidiana e melhora em sua reinserção social.

Apesar da importância do repertório de habilidades sociais dos familiares cuidadores do paciente psiquiátrico, poucos estudos foram realizados para caracterizá-las. Em uma busca feita nas bases de dados Scientific Electronic Library Online (SciELO), Lilacs, Index Psi Periódicos Técnico-Científicos (IndexPsi), Medical Literature Analysis and Retrieval System Online (Medline), Psycinfo, Pubmed e ISI Web of Knowledge, foram encontrados apenas dois estudos nessa temática (Fernandes, Luiz, Miyazaki, \& Marques Filho, 2009; Robinson, 1990). A pesquisa de Fernandes et al. (2009) avaliou um programa de orientação para familiares cuidadores de crianças com transtornos psiquiátricos. O estudo de Robinson (1990) investigou as relações entre assertividade, suporte social, autoestima e sobrecarga de familiares cuidadores de idosos com demência. No entanto, nenhum dos dois estudos caracterizou o repertório de habilidades sociais dos familiares cuidadores, o que representa uma lacuna na literatura da área.

Nesse sentido, o presente estudo teve como objetivos: 1) investigar o grau de habilidades sociais e, em particular, da subclasse de assertividade, dos familiares cuidadores de pacientes psiquiátricos, 2) identificar os subgrupos que apresentam maiores escores dessas habilidades; 3) avaliar a relação entre o grau de habilidades sociais e o uso de estratégias de enfrentamento.

\section{Método}

\section{Participantes}

A amostra foi constituída por familiares cuidadores de pacientes psiquiátricos, atendidos no Centro de Atenção Psicossocial (CAPS) de uma cidade de Minas Gerais, que preenchiam os critérios de inclusão e exclusão adotados. Foram incluídos os familiares com 18 anos ou mais, de ambos os gêneros, indicados pela coordenadora do CAPS como os principais cuidadores dos pacientes e que conviviam diariamente com eles. Foram incluídos familiares cuidadores de pacientes com diagnóstico do espectro da Esquizofrenia (F20-F29) ou Transtornos do Humor e Afetivos (F30-F39), conforme a Classificação Internacional de Doenças (CID-10) da OMS (1998), apontados nos prontuários do serviço. Foram excluídos os familiares com algum transtorno psiquiátrico, segundo informações do serviço, ou com dificuldades em compreender os itens dos instrumentos de medida.

Dentre os familiares listados nos prontuários do serviço, 83 preencheram os critérios de inclusão e exclusão. Decidiu-se avaliar toda essa população-alvo e não apenas uma amostra, pois não se tratava de um número excessivo de sujeitos. Dentre eles, dez não foram encontrados devido a problemas de endereço e 17 recusaram-se a participar. Foram realizadas 56 entrevistas. Foram eliminados três familiares, por não compreenderem os instrumentos de medida. Assim, a amostra final envolveu 53 familiares.

\section{Instrumentos}

Inventário de Habilidades Sociais (IHS-DelPrette): trata-se de um inventário que possui qualidades psicométricas satisfatórias de validade e fidedignidade (Z.A.P. Del Prette, Del Prette, \& Barreto, 1998; Z.A.P. Del Prette \& Del Prette, 2001; Bandeira, Costa, Z.A.P. Del Prette, Del Prette, \& Gerk-Carneiro, 2000). Possui 38 itens, que descrevem ações ou sentimentos diante de situações sociais. O respondente deve indicar a frequência com que age ou sente 
daquela maneira em cada situação. As alternativas de resposta são dispostas em uma escala Likert de cinco pontos, que varia de "nunca ou raramente" a "sempre ou quase sempre". O inventário possui cinco subescalas, que avaliam as habilidades referentes a: enfrentamento e autoafirmação com risco, autoafirmação na expressão de sentimento positivo, conversação e desenvoltura social, autoexposição a desconhecidos, e situações novas e autocontrole da agressividade.

Rathus Assertiveness Schedule (RAS, Escala de Assertividade Rathus): Essa escala foi traduzida e adaptada para o Brasil por Pasquali e Gouveia (1990) e possui qualidades psicométricas satisfatórias de validade e fidedignidade (Pasquali \& Gouveia, 1990; Pasquali, Moura, \& Freitas, 2002). É composta por 30 itens, que apresentam afirmativas sobre os comportamentos das pessoas em situações cotidianas. Os respondentes devem indicar o quanto essas descrições são parecidas com eles. As alter-nativas de resposta são distribuídas em uma escala do tipo Likert de seis pontos, que varia de "não descreve absolutamente nada" a "descreve per-feitamente". A escala inclui um fator geral e três subescalas, que avaliam os graus de timidez, agres-sividade e assertividade.

Questionário sociodemográfico e clínico: para caracterizar a amostra, foi utilizado um questionário com questões sobre as variáveis sociodemográficas dos familiares e pacientes, assim como as variáveis clínicas destes últimos. No questionário, foram incluídas questões sobre estratégias para lidar com os pacientes e a subescala 4 da Escala Modos de Enfrentamento de Problemas (EMEP), referente à busca de suporte social (Seidl, Tróccoli, \& Zannon, 2001). O instrumento foi testado quanto a sua adequabilidade e facilidade de compreensão.

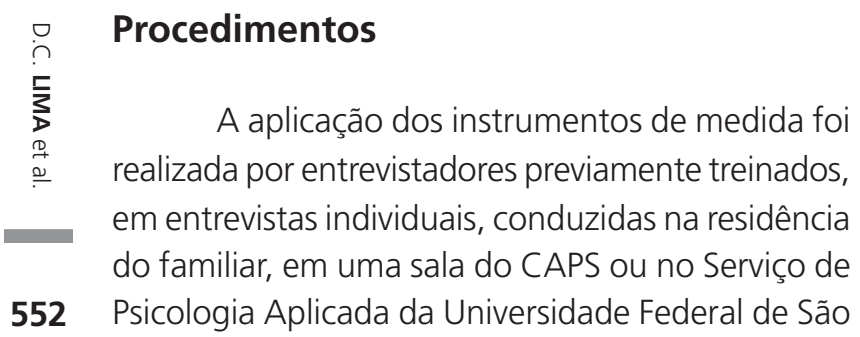

João del-Rei, conforme a disponibilidade dos sujeitos. A participação de todos foi voluntária.

Este estudo foi aprovado pela coordenação do Caps e pela Comissão de Ética em Pesquisas Envolvendo Seres Humanos da Universidade de origem dos autores (Processo no 007/2012, aprovado em 19 de março de 2012). Os objetivos do estudo foram informados a todos os participantes. O sigilo das informações foi assegurado, assim como os familiares foram informados de que sua participação não ocasionaria riscos ou desconfortos. Foi solicitado que os participantes assinassem um Termo de Consentimento Livre e Esclarecido.

Os dados foram analisados por meio do programa informatizado Statistical Package for Social Sciences (SPSS), versão 13.0. Para descrição da amostra, foram utilizadas estatísticas descritivas de cálculos de média, desvios-padrão e porcentagens. Para identificar subgrupos de familiares com maiores e menores escores no IHS-Del-Prette e na RAS, foi utilizado o teste $t$ de Student para amostras independentes. O teste de correlação de Pearson foi utilizado para verificar a correlação dos escores obtidos no IHS-Del-Prette e na RAS com outras variáveis investigadas.

\section{Resultados}

\section{Descrição da amostra}

A idade média dos familiares cuidadores era de 48,64 anos (Desvio-Padrão-DP = 15,15), com variação de 18 a 79 anos. A maioria era do gênero feminino $(75,5 \%)$, casada $(37,7 \%)$, com ensino fundamental incompleto $(50,9 \%)$. No que se refere ao nível socioeconômico, $75,5 \%$ dos familiares possuíam renda própria, com valor mensal médio de $\mathrm{R} \$ 901,56$. Em relação ao parentesco, 34,0\% dos familiares eram pais ou mães dos pacientes e $35,8 \%$ eram irmãos.

Por sua vez, os pacientes tinham média de idade de 41,36 anos ( $D P=10,23$ ), com idade mínima de 20 anos e máxima de 62 anos. A maioria era do sexo feminino $(54,70 \%)$, solteira $(69,89 \%)$, com 
ensino fundamental incompleto (52,80\%) e possuía renda própria $(73,60 \%)$, com valor mensal médio de $R \$ 608,35$. No que se refere às categorias diagnósticas, 69,80\% deles tinham diagnóstico de Esquizofrenia, Transtornos Esquizotípicos e Transtornos Delirantes, enquanto 30,20\% apresentavam Transtornos do Humor e Afetivos, segundo Classificação da CID-10 (OMS, 1998).

\section{Análise dos escores do IHS-Del-Prette e da RAS}

Foram calculadas as médias, DP, e mínimos e máximos dos escores obtidos pelos familiares cuidadores no IHS-Del-Prette e na RAS. Esses dados estão apresentados na Tabela 1.

A partir dos escores obtidos, foi possível identificar as porcentagens de familiares que se encontravam abaixo da média da amostra. Para o escore global, $27(52,94 \%)$ dos familiares cuidadores estavam abaixo da média. Para as subescalas, 0 número de familiares abaixo da média foi de 28 (52,80\%), 26 (49,06\%), 30 (56,60\%), 27 (50,94\%) e $22(41,51 \%)$, nas subescalas de 1 a 5 do IHS, respectivamente. Esses resultados indicaram que, com exceção das subescalas 2 e 5, mais de 50,00\% dos familiares cuidadores obtiveram escores abaixo da média.

Em relação à escala RAS, os resultados possibilitaram identificar que 26 familiares $(49,06 \%)$ estavam acima da média encontrada para o grau de inibição, 30 familiares (56,60\%) estavam acima da média do grau de timidez, e 25 familiares (47,17\%) acima da média do grau de agressividade. No que se refere à avaliação da assertividade, no sentido de fazer valer seus direitos, 23 familiares (43,40\%) se situaram abaixo da média encontrada.

\section{Análises das variáveis associadas aos escores do IHS-Del-Prette}

Na Tabela 2, são apresentados os resultados das relações significativas entre o grau de habilidades sociais avaliadas pelo IHS-Del-Prette e as variáveis sociodemográficas e estratégias de enfrentamento. As variáveis sociodemográficas estado civil, renda e idade também foram analisadas, porém não apresentaram diferença ou correlação estatisticamente significativa.

Para a variável escolaridade, houve diferenças estatisticamente significativas entre as categorias, para o escore global de habilidades sociais $(t=-3,68 ; p=0,001)$ e para as subescalas $1(t=-2,37$; $p=0,022), 3(t=-4,25 ; p<0,001)$ e $4(t=-3,54$; $p=0,001$ ), sendo que os familiares com ensino médio completo apresentaram escores mais elevados. Em relação à variável sexo, foi encontrado que os homens apresentaram escores mais elevados $(t=2,74 ; p=0,008)$ para a subescala 5 do

\section{Tabela 1}

Médias, desvios-padrão e valores mínimo e máximo dos escores globais e das subescalas do IHS-Del-Prette e da RAS

\begin{tabular}{|c|c|c|c|c|c|}
\hline Instrumento/Escore global ou subescala & $n$ & Média & DP & Mínimo & Máximo \\
\hline \multicolumn{6}{|l|}{ IHS-Del-Prette } \\
\hline Escore global & 51 & 90,00 & 20,03 & 54,00 & 141,00 \\
\hline Enfrentamento e autoafirmação com risco & 53 & 8,53 & 3,57 & 3,04 & 17,70 \\
\hline Autoafirmação na expressão do afeto positivo & 53 & 9,16 & 1,66 & 5,69 & 11,70 \\
\hline Conversação e desenvoltura social & 53 & 6,17 & 2,41 & 0,94 & 11,02 \\
\hline Autoexposição a desconhecidos e situações novas & 53 & 2,78 & 1,37 & 0,00 & 5,51 \\
\hline Autocontrole da agressividade em situações aversivas & 53 & 1,07 & 0,77 & $-0,93$ & 2,47 \\
\hline \multicolumn{6}{|l|}{ RAS } \\
\hline Escore global & 53 & 67,34 & 14,55 & 38,00 & 96,00 \\
\hline Timidez & 53 & 41,74 & 10,90 & 19,00 & 65,00 \\
\hline Agressividade & 53 & 15,58 & 4,74 & 6,00 & 27,00 \\
\hline Assertividade & 53 & 29,43 & 6,55 & 15,00 & 42,00 \\
\hline
\end{tabular}


Tabela 2

Análises das variáveis sociodemográficas e das estratégias de enfrentamento em relação aos escores de habilidades sociais

\begin{tabular}{|c|c|c|c|c|c|c|}
\hline Variáveis & Escore global ou subescala & Categoria & Média & DP & $r$ & $t / p$ \\
\hline \multirow[t]{8}{*}{ Escolaridade } & \multirow[t]{2}{*}{ IHS Global } & Ensino médio incompleto & 83,18 & 16,85 & & $-3,68$ \\
\hline & & Ensino médio completo & 102,50 & 19,76 & & $0,001^{*}$ \\
\hline & \multirow[t]{2}{*}{ IHS S1 } & Ensino médio incompleto & 7,73 & 3,01 & & $-2,37$ \\
\hline & & Ensino médio completo & 10,08 & 4,12 & & $0,022^{*}$ \\
\hline & \multirow[t]{2}{*}{ IHS S3 } & Ensino médio incompleto & 5,29 & 2,09 & & $-4,25$ \\
\hline & & Ensino médio completo & 7,87 & 2,09 & & $0,000^{* * *}$ \\
\hline & \multirow[t]{2}{*}{ IHS S4 } & Ensino médio incompleto & 2,35 & 1,22 & & $-3,54$ \\
\hline & & Ensino médio completo & 3,62 & 1,27 & & $0,001^{* *}$ \\
\hline \multirow[t]{2}{*}{ Sexo } & \multirow[t]{2}{*}{ IHS S5 } & Masculino & 1,55 & 0,58 & & 2,74 \\
\hline & & Feminino & 0,92 & 0,76 & & $0,008^{* *}$ \\
\hline \multirow[t]{2}{*}{ Trabalho } & \multirow[t]{2}{*}{ IHS S4 } & Não & 2,31 & 1,28 & & $-2,96$ \\
\hline & & Sim & 3,36 & 2,27 & & $0,005^{* *}$ \\
\hline \multirow[t]{2}{*}{ Estratégias para lidar com o paciente } & IHS Global & & & & 0,29 & $0,041^{*}$ \\
\hline & IHS S2 & & & & 0,41 & $0,002^{* *}$ \\
\hline \multirow[t]{4}{*}{ Busca por suporte social(EMEP F4) } & IHS Global & & & & 0,33 & $0,019^{*}$ \\
\hline & IHS S1 & & & & 0,31 & $0,024^{*}$ \\
\hline & IHS S2 & & & & 0,36 & $0,007^{* *}$ \\
\hline & IHS S3 & & & & 0,30 & $0,029^{*}$ \\
\hline
\end{tabular}

Nota: ${ }^{*} p<0,05 ;{ }^{* *} p<0,01 ;{ }^{* * *} p<0,001$.

DP: Desvio-Padrão; IHS S1, IHS S2, IHS S3, IHS S4 e IHS S5: Subescalas 1 a 5 do Inventário de Habilidades Sociais (IHS-Del-Prette); EMEP: Escala Modos de Enfrentamento de Problemas.

IHS-Del-Prette (autocontrole da agressividade em situações aversiva). No que se refere à variável trabalho, observou-se que os familiares que trabalhavam apresentaram escores mais elevados do que os que não trabalhavam, com relação à subescala 4 do IHS-del-Prette $(t=-2,96 ; p=0,005)$ (autoexposição a desconhecidos e situações novas) e à subescala 2 da RAS ( $t=-3,23 ; p=0,002)$ (agressividade).

As análises correlacionais mostraram resultados positivos significativos entre o número de estratégias usadas pelos familiares para lidar com os pacientes e dois escores do IHS-Del-Prette: o escore global $(r=0,29, p=0,041)$ e o escore obtido na subescala $2(r=0,41, p=0,002)$. Esse resultado indicou que, quanto maior era o grau de habilidades sociais dos familiares (global e na subescala 2), maior era o número de estratégias de enfrentamento utilizadas para lidar com o paciente.

Foram encontradas, ainda, correlações positivas significativas entre o grau de habilidades sociais e as estratégias de busca por suporte social (subescala 4 da EMEP), para o escore global $(r=0,33$, $p=0,019)$ e para as subescalas $1(r=0,31, p=0,024)$,

$5542(r=0,36, p=0,007)$ e $3(r=0,30, p=0,029)$ do
IHS-Del-Prette. Quanto mais elevados eram esses escores, maior era a frequência de utilização, pelos familiares, de busca por suporte social.

\section{Análises das variáveis associadas aos escores da RAS}

Na Tabela 3, são apresentados os resultados das relações entre os escores obtidos na escala RAS e as variáveis sociodemográficas, que foram estatisticamente significativas. Outras variáveis como sexo e trabalho também foram analisadas, mas não apresentaram diferenças estatisticamente significativas. Foi analisada a relação dos escores da RAS com as estratégias para lidar com os pacientes e a busca por suporte social (subescala 4 da EMEP), porém sem resultado estatisticamente significativo.

Com relação à escolaridade, os resultados da Tabela 3 mostram que os familiares cuidadores com ensino médio incompleto apresentaram maior grau de inibição $(t=3,78 ; p<0,001)$ (escore global da RAS) e de timidez ( $t=3,69 ; p=0,001$ ) (subescala 1 da RAS). Já os familiares com ensino médio com- 
Tabela 3

Análise das variáveis sociodemográficas em relação aos escores da escala RAS

\begin{tabular}{|c|c|c|c|c|c|c|}
\hline Variáveis & Escore global ou subescala & Categoria & Média & DP & $r$ & $t / p$ \\
\hline \multirow[t]{6}{*}{ Escolaridade } & \multirow[t]{2}{*}{ RAS global } & Ensino médio incompleto & 72,17 & 13,50 & & 3,78 \\
\hline & & Ensino médio completo & 57,94 & 11,89 & & $0,000^{* * *}$ \\
\hline & \multirow[t]{2}{*}{ RAS S1 } & Ensino médio incompleto & 45,29 & 10,01 & & 3,69 \\
\hline & & Ensino médio completo & 34,83 & 9,27 & & $0,001^{\star *}$ \\
\hline & \multirow[t]{2}{*}{ RAS S2 } & Ensino médio incompleto & 14,26 & 4,53 & & $-3,07$ \\
\hline & & Ensino médio completo & 18,17 & 4,12 & & $0,003^{* *}$ \\
\hline \multirow[t]{2}{*}{ Estado civil } & \multirow[t]{2}{*}{ RAS global } & Solteiro, divorciado ou viúvo & 71,06 & 14,74 & & 2,21 \\
\hline & & Casados ou vivendo como casados & 62,48 & 13,04 & & $0,032^{*}$ \\
\hline \multirow[t]{2}{*}{ Trabalho } & \multirow[t]{2}{*}{ RAS S2 } & Não & 13,83 & 4,39 & & $-3,23$ \\
\hline & & $\operatorname{sim}$ & 17,71 & 4,32 & & $0,002^{* *}$ \\
\hline Idade & RAS S2 & & & & $-0,27$ & $0,050^{*}$ \\
\hline
\end{tabular}

Nota: ${ }^{*} p<0,05 ;{ }^{* *} p<0,01 ;{ }^{* * *} p<0,001$.

RAS: Rathus Assertiveness Schedule; RAS S1: Subescala 1; RAS S2: Subescala 2; DP: Desvio-Padrão.

pleto apresentaram maior grau de agressividade $(t=-3,07 ; p=0,003)$ (subescala 2 da RAS).

Para o estado civil, foi encontrado que os familiares casados ou que viviam como casados apresentaram menor grau de inibição $(t=2,74$; $p=0,008$ ) do que os solteiros, divorciados ou viúvos.

Em relação ao trabalho, observou-se que os familiares que trabalhavam apresentavam maior grau de agressividade $(t=-3,23 ; p=0,002)$ do que aqueles que não trabalhavam (subescala 2 da RAS).

Em relação à idade, obteve-se uma correlação negativa significativa $(r=-0,271, p=0,05)$ entre o grau de agressividade e a idade do cuidador.

\section{Discussão}

Os resultados do presente estudo possibilitaram identificar os escores dos familiares cuidadores de pacientes psiquiátricos em relação às habilidades sociais, avaliadas pelo IHS-Del-Prette, e à subclasse de assertividade, avaliada pela RAS. Apesar de não ser possível utilizar os dados normativos de classificação dos instrumentos, uma vez que eles foram elaborados com outras populações, foi possível identificar que mais de $50 \%$ dos familiares cuidadores estavam abaixo da média geral para o escore global e para as subescalas 1 (enfrentamento e autoafirmação com risco), 3 (conversação e desenvoltura social) e 4 (autoexposição a desconhecidos e situações novas) do IHS-Del-Prette. Para as subes- calas 2 (autoafirmação na expressão de afeto positivo) e para a subescala 5 (autocontrole da agressividade em situações aversivas), mais de $40 \%$ dos familiares estavam abaixo da média. Em relação à escala RAS, porcentagens elevadas de familiares cuidadores estavam acima da média no que se refere ao grau de inibição (mais de 45\%), de timidez (mais de 55\%) e de agressividade (mais de 45\%). Além disso, uma porcentagem elevada estava abaixo da média (mais de $45 \%$ ) para o grau de assertividade, no sentido de fazer valer os seus direitos.

Esses resultados são preocupantes quando discutidos com base na literatura. No modelo teórico explicativo da sobrecarga, elaborado por Maurin e Boyd (1990), a qualidade do relacionamento entre familiares e pacientes, o suporte social recebido pelos familiares e as estratégias de enfrentamento que eles utilizam são fatores moduladores que podem diminuir o grau de sobrecarga. Como todos esses fatores dependem das habilidades sociais, os cuidadores com dificuldade nessas habilidades podem apresentar níveis mais altos de sobrecarga.

Gotlieb (1981) destacou que as habilidades sociais são essenciais na construção de interações sociais adequadas e no desenvolvimento de redes de suporte social. Problemas de repertório em habilidades sociais e, mais especificamente, na subclasse de assertividade podem dificultar a interação dos familiares com os pacientes, no manejo dos comportamentos problemáticos na vida cotidiana e podem comprometer o desenvolvimento de uma rede de 
suporte social para ajudá-los nos cuidados aos pacientes. Além disso, os familiares com dificuldades nas habilidades sociais podem constituir modelos pobres como agentes educativos para os pacientes.

Os resultados indicaram, ainda, relações significativas entre os menores escores de habilidades sociais do IHS-Del-Prette e algumas variáveis sociodemográficas, tais como menor escolaridade, sexo feminino e não ter um trabalho. Na literatura consultada, não foram encontrados estudos que tenham analisado os fatores sociodemográficos dos familiares cuidadores em relação às habilidades sociais. No entanto, estudos realizados com outras populações encontraram resultados semelhantes ao do presente trabalho. Em estudo realizado com uma amostra de bombeiros, um menor nível de escolaridade esteve relacionado a um menor escore global de habilidades sociais, avaliado pelo IHS-Del-Prette (Oliveira, 2010). Em relação à variável sexo, um estudo realizado com dependentes e não dependentes de álcool encontrou que os participantes do sexo feminino apresentaram escores mais baixos em relação ao controle da agressividade em situações aversivas (subescala 5 do IHS-Del-Prette) (Aliane, Lourenço, \& Ronzani, 2006).

A análise dos escores da escala RAS indicou que os familiares cuidadores com ensino médio incompleto eram mais inibidos (escore global) e tímidos (subescala 1). Por outro lado, os familiares cuidadores com ensino médio completo apresentaram maior grau de agressividade (subescala 2). Em relação ao estado civil, os familiares cuidadores solteiros, divorciados ou viúvos eram mais inibidos (escore global). No que se refere à idade e a ter ou não um trabalho, os familiares cuidadores mais novos e que trabalhavam apresentaram maior grau de agressividade (subescala 2). Na literatura consultada, não foram encontrados estudos que avaliaram as variáveis sociodemográficas em relação ao escore global ou subescalas da RAS.

Os resultados referentes às estratégias de enfrentamento mostraram que os familiares cuidadores com escores mais elevados na subescala 2 do IHS-Del-Prette (autoafirmação na expressão do 556 afeto positivo) e no escore global desse instrumento apresentaram maior número de estratégias para lidar com os pacientes. Não foram encontrados estudos que avaliassem essas estratégias em familiares cuidadores de pacientes. No entanto, um estudo (Miguel \& Bueno, 2011) que investigou as relações entre habilidades sociais e estratégias de enfrentamento, em adultos, encontrou correlação positiva entre a subescala 2 do IHS-Del-Prette e a subescala de estratégia de enfrentamento focalizada no problema da EMEP. No presente trabalho, foi encontrado que os familiares cuidadores com escores mais elevados nas subescalas de enfrentamento e autoafirmação com risco (subescala 1), autoafirmação na expressão de afeto positivo (subescala 2) conversação e desenvoltura social (subescala 3) e no escore global do IHS-Del-Prette apresentaram escores mais elevados na busca por suporte social (subescala 4 da EMEP). Esse último achado confirma o resultado encontrado no estudo de Robinson (1990), em que as habilidades sociais de familiares cuidadores de pacientes com demência correlacionaram, de maneira positiva e significativa, com a busca por suporte social.

Apesar do cuidado e rigor metodológico utilizado, o presente estudo apresentou algumas limitações. Primeiro, embora a pesquisa tenha sido realizada com todos os familiares cuidadores da população-alvo que preenchiam os critérios de inclusão e exclusão, foi incluído apenas um serviço de saúde mental, limitando assim a generalização dos resultados. Em segundo lugar, apesar de os instrumentos de medida utilizados apresentarem qualidades psicométricas satisfatórias, eles não são específicos para esta população-alvo.

Os resultados encontrados forneceram importantes informações sobre o repertório de habilidades sociais e da subclasse de assertividade dos familiares cuidadores. São informações relevantes para a avaliação dos serviços de saúde mental, pois se recomenda que eles ofereçam intervenções eficazes para que os familiares conheçam melhor a problemática do paciente e aprendam a lidar com os seus comportamentos na vida cotidiana (OMS, 2001). As habilidades avaliadas neste estudo podem contribuir para a qualidade do relacionamento dos familiares com o paciente, o que constitui um dos 
fatores moduladores da sobrecarga do cuidador, afetando assim a qualidade dos cuidados que ele presta.

Os resultados indicaram a necessidade de serem desenvolvidos programas específicos de Treinamento de Habilidades Sociais (THS) para essa população-alvo, nos serviços de saúde mental. Uma melhora do repertório de habilidades sociais dos familiares cuidadores, resultante desse tipo de treinamento, poderá aumentar a qualidade de seu relacionamento com o paciente, potencializando os cuidados prestados e a própria qualidade de vida deste último.

Sugere-se que futuros estudos possam elaborar instrumentos específicos de avaliação das habilidades sociais para essa população-alvo, enfatizando as contingências sociais próprias que se apresentam no seu relacionamento com o paciente. Além disso, também são necessários estudos com familiares cuidadores de pacientes de outros serviços de saúde mental. Será igualmente pertinente que estudos futuros investiguem a relação entre as habilidades sociais dos cuidadores e as variáveis referentes ao grau de ansiedade e depressão, tendo em vista que o nível de sobrecarga vivenciada por eles pode afetar sua saúde mental.

\section{Referências}

Abelha, L., Muñoz, M. D., Gonçalves, S., Fagundes, P., Barbosa, D. R., \& Legay, L. F. (2006). Evaluation of social disablement, psychiatric symptoms and autonomy in long-stay psychiatric patients. Revista de Psiquiatria Clínica, 33(1), 10-17.

Aliane, P. P., Lourenço, L. M., \& Ronzani, T. M. (2006). Estudo comparativo das habilidades sociais de dependentes e não dependentes de álcool. Psicologia em Estudo, 11(1), 83-88.

Bandeira, M. (2003). Avaliando a competência social de pacientes psiquiátricos: Questões conceituais e metodológicas. In A. Del Prette \& Z. A. P. Del Prette (Orgs.), Habilidades sociais: desenvolvimento e aprendizagem: questões conceituais, avaliação e intervenção (pp.207-234). Campinas: Alínea.

Bandeira, M., Costa, M. N., Del Prette, Z. A. P., Del Prette, A., \& Gerk-Carneiro, E. (2000). Qualidades psicométricas do inventário de habilidades sociais: estudo sobre a estabilidade temporal e a validade concomitante. Estudos de Psicologia, 5(2), 401-419.
Barroso, S., Bandeira, M., \& Nascimento, E. (2007). Sobrecarga de familiares de pacientes psiquiátricos atendidos na rede pública. Revista de Psiquiatria Clínica, 34(6), 270-277.

Barroso, S., Bandeira, M., \& Nascimento, E. (2009). Fatores preditores da sobrecarga subjetiva de familiares de pacientes psiquiátricos atendidos na rede pública de Belo Horizonte, Minas Gerais, Brasil. Caderno de Saúde Pública, 25(9), 1957-1968.

Bartels, S. J., Forester, B., Mueser, K. T., Miles, K. M., Dums, A. R., Pratt, S. I., ... White, P. (2004). Enhanced skills training and health care management for older persons with severe mental illness. Community Mental Health Journal, 40(1), 75-90.

Del Prette, Z. A. P., \& Del Prette, A. (1999). Psicologias das habilidades sociais: terapia e educação. Petrópolis: Vozes.

Del Prette, Z. A. P., \& Del Prette, A. (2001). Inventário de Habilidades Sociais (IHS-Del-Prette): manual de aplicação, apuração e interpretação. São Paulo: Casa do Psicólogo.

Del Prette, Z. A. P., \& Del Prette, A. (2008). Um sistema de categorias de habilidades sociais educativas. Paidéia, 18(4), 517-530.

Del Prette, Z. A. P., Del Prette, A., \& Barreto, M. C. M. (1998). Análise de um Inventário de Habilidades Sociais (IHS-Del-Prette) em uma amostra de universitários. Psicologia: Teoria e Pesquisa, 14(3), 219-228.

Fernandes, L. F. B., Luiz, A. M. A. G., Miyazaki, M. C. O. S., \& Marques Filho, A. B. (2009). Efeitos de um programa de orientação em grupo para cuidadores de crianças com transtornos psiquiátricos. Estudos de Psicologia (Campinas), 26(2), 147-158.

Gotlieb, B. (1981). Preventing interventions involving social networks and social support. In B. Gotlieb (Org.). Social Networks and Social Support Sage Studies in Community Mental Health (Vol.4, pp.201-232). Beverly Hills: Sage.

Loukissa, A. D. (1995). Family burden in chronic mental illness: A review of research studies. Journal of Advanced Nursing, 21(2), 248-255.

Maurin, J. T., \& Boyd, C. B. (1990). Burden of mental illness on the family: A critical review. Archives of Psychiatric Nursing, 4(2), 99-107.

Miguel, S. P., \& Bueno, M. A. (2011). Habilidades sociais e estratégias de enfrentamento: um estudo correlacional. Psicologia. PT: O Portal dos Psicólogos. Recuperado em janeiro 7, 2013, de psicologia.pt/ artigos/textos/A0578.pdf

Oliveira, P. A. (2010). Habilidades sociais, depressão, ansiedade e alcoolismo em bombeiros: Um estudo correlacional (Dissertação de mestrado não-publicada). Universidade Federal de São Carlos. 
Organização Mundial de Saúde. (1998). CID-10: Classificação Internacional de Doenças e Problemas Relacionados à Saúde (10 ed.). São Paulo: Edusp.

Organização Mundial de Saúde. (2001). Relatório mundial da saúde: saúde mental: nova concepção, nova esperança. Lisboa: OMS.

Organização Mundial de Saúde. (2008). Integração de saúde mental nos cuidados de saúde primária: uma perspectiva global. Lisboa: OMS.

Pasquali, L., \& Gouveia, V. V. (1990). Escala de Assertividade Rathus-RAS: adaptação brasileira. Psicologia: Teoria e Pesquisa, 6(3), 233-249.

Pasquali, L., Moura, C. F., \& Freitas, L. C. O. (2002). Escala de Assertividade Rathus - RAS. Brasília: LabPAM.

Patterson, T. L., \& Mausbach, B. T. (2010). Measurement of functional capacity: A new approach to understanding functional differences and real-world behavioral adaptation in those with mental illness. Annual Review of Clinical Psychology, 27(6), 139-154.

Robinson, K. (1990). The relationships between social skills, social support, self-esteem and burden in adult caregivers. Journal of Advanced Nursing, 15(7), 788-795.

Rose, L. E. (1996). Families of psychiatric patients: A critical review and future research directions. Archives of Psychiatric Nursing, 10(2), 67-76.
Seidl, E. M. F., Tróccoli, B.T., \& Zannon, C.M.L.C. (2001). Análise fatorial de uma medida de estratégias de enfrentamento. Psicologia: Teoria e Pesquisa, 17(3), 225-234.

Soares, C. B., \& Munari, D. B. (2007). Considerações acerca da sobrecarga em familiares de pessoas com transtornos mentais. Ciência, Cuidado e Saúde, 6(3), 357-362.

Tessler R. C., \& Gamache G. M. (2000). Family Experiences with mental illness. Westport: Auburn House.

Thornicroft, G., \& Tansella, M. (2010). Boas práticas em saúde mental comunitária. Barueri: Manole.

Trieman, F., \& Leff, J. (2002). Long-term outcome of long-stay psychiatric in-patients considered unsuitable to live in the community. The TAPS project 44. British Journal of Psychiatry, 181, 429-432.

Vidal, C. E. L., Bandeira, M., \& Gontijo, E. D. (2008). Reforma psiquiátrica e serviços residenciais terapêuticos. Jornal Brasileiro de Psiquiatria, 57(1), 70-79.

Recebido em: 12/4/2013

Versão final em: 2/9/2013

Aprovado em: 23/9/2013 